\title{
Relation between the Intervals $\Delta E$ and $\Delta t$ Obtained in the De-Excitation Process of Electrons in Metals
}

\author{
Stanisław Olszewski \\ Institute of Physical Chemistry, Polish Academy of Sciences, Warsaw, Poland \\ Email: olsz@ichf.edu.pl \\ Received November 30, 2011; revised January 6, 2012; accepted January 15, 2012
}

\begin{abstract}
A relation between the intervals of energy and time, derived in a former paper and associated with the electron transitions on the Fermi surface of a metal, is examined in comparison with the experimental data. These data are obtained from the de-excitation process of electrons in metals. A comparison between theory and experiment demonstrated that the new relation between energy and time is fitted much better for the experimental results than the well-known relation due to the Heisenberg theory.
\end{abstract}

Keywords: De-Excitation of Electrons; Metals; Relations between the Intervals of Energy and Time

\section{Introduction}

A well-known relation between the intervals of energy and time, deduced by Heisenberg [1,2], namely

$$
\Delta E \Delta t>\hbar
$$

is often considered as analogous to the uncertainty relation represented by the intervals of the particle position and momentum. However, it has been stressed a time ago that the significance of (1) is entirely different than that of the formula

$$
\delta x_{i} \delta p_{i}>\hbar
$$

where $i=1,2,3$ label the coordinates of the position and momentum in a three-dimensional space. In fact (1) concerns the exactly measured intervals of energy and time, whereas (2) refers to the uncertainties of the values of the position and momentum coordinates measured at the same instant of time [3-5]. Nevertheless, the uncertainty relation for energy and time similar to (2) can be also derived [5].

The aim of the present paper is to give a kind of a new look on the relation between $\Delta E$ and $\Delta t$ done from both the theoretical and experimental point of view. In fact the formula in (1) is not a unique proposal of the coupling connecting $\Delta E$ and $\Delta t$. An alternative formula can be derived when the electron transitions in the electron gas are effectuated in the field of the magnetic induction $\boldsymbol{B}$. Let us assume that $\boldsymbol{B}$ is directed along axis $z$, so $B=B_{z}$, and simultaneously the limitations of the electron velocity imposed by the special theory of relativity are also taken into account. In this case a condition satisfied by the change of the momentum square
$k_{F}^{2}$ at the Fermi surface within the time interval $\Delta t$ becomes [6]:

$$
\left(k_{F}^{2}-k_{z}^{2}\right)(\Delta t)^{2}>\frac{1}{c^{2}}
$$

The momentum change in (3) can be referred to that of energy by multiplying the both sides of (3) by the term

$$
\hbar^{2} c^{2}
$$

For, this operation gives in place of (3) the relation

$$
2 m c^{2} \Delta E(\Delta t)^{2}>\hbar^{2}
$$

if we note the well-known free-electron formula for the change of the Fermi energy:

$$
\Delta E=\frac{\hbar^{2}}{2 m}\left(k_{F}^{2}-k_{z}^{2}\right)
$$

In effect, when instead of (5) the square-root of the both sides of this formula is taken into account, we obtain a different relation between $\Delta E$ and $\Delta t$ than (1):

$$
(2 m)^{1 / 2} c(\Delta E)^{1 / 2} \Delta t>\hbar
$$

A characteristic point is that (7) does not contain $B$, although some limitation for the maximal value of this parameter and, consequently, the cyclotron frequency $\Omega_{0}$ induced in the electron gas, is imposed by the theory [6].

The lack of $B$ makes (7) a competitive expression to (1). Sections 2 and 3 try to clarify this competition on the experimental basis.

\section{Experimental Approach}

This approach is based on the de-excitaion process of the 
photoelectrons [7]. In considering the decay of the motion of a photoelectron excited originally from the freeelectron gas we have the notion of the lifetime of that electron in its excited state

$$
\tau_{e}=\Delta t
$$

and a reference of this lifetime to the electron mean free path

$$
l_{e}=\tau_{e} v_{g}
$$

$v_{g}$ is the group velocity of a photoelectron in its excited state.

The amount of energy lost by the excited electron of energy $E$ in the de-excitation process is most often in the range of magnitude

$$
\Delta E=\frac{1}{2}\left(E-E_{F}\right)=\frac{m}{4}\left(v_{g}^{2}-v_{F g}^{2}\right)
$$

$v_{F g}$ is the group velocity of an electron on the Fermi level. The relation in (10) holds because, due to the interaction of an excited electron with a less energetic electron, the photon energy is shared between two excited electrons [7].

In case a collective motion of the conduction electrons is taken into account, the plasmon scattering from the interaction of the excited electron with a set of conduction electrons should be considered. In this circumstanc $l_{e}$ in (9) is modified into

$$
l_{e}^{\mathrm{coll}}=\frac{l_{p e} l_{e e}}{l_{p e}+l_{e e}}
$$

Here $l_{p e}$ is coming from the plasmon scattering and $l_{e e}$ from the electron-electron scattering [7].

A characteristic experimental result $[7,8]$ is that for large $E$ the length $l_{e}^{\text {coll }}$ which replaces $l_{e}$ in (9) tends approximately to a constant value independent of $E$. This result substituted to (9) provides us with the relation

$$
\frac{l_{e}^{\text {coll }}}{\tau_{e}} \sim \frac{1}{\tau_{e}} \sim v_{g}
$$

Another relation for the excited electron is its group velocity

$$
v_{g} \cong m^{-1 / 2}(2 E)^{1 / 2}
$$

because of the well-known formula for the kinetic energy. At the end of the de-excitation process the electron is close to the Fermi level, so its velocity is decreased to

$$
v_{F g} \cong m^{-1 / 2}\left(2 E_{F}\right)^{1 / 2}
$$

In many excited cases, for example in the Auger effect where $E$ of few hundreds of $\mathrm{eV}$ are involved, we have

$$
\Delta E=\frac{1}{2}\left(E-E_{F}\right) \approx \frac{1}{2} E
$$

because $E_{F}$ is so small that it can be approximately neglected in comparison with large $E$. For example, in the metallic Cs examined in the photoexcitation process, $E_{F}$ is smaller than $1.6 \mathrm{eV}$ [9]. Consequently, due to (8), (13) and (15):

$$
l_{e}^{\text {coll }}=\tau_{e} v_{g} \approx 2 m^{-1 / 2}(\Delta E)^{1 / 2} \Delta t
$$

because $2 E$ in (13) is approximately equal to $4 \Delta E$.

A characteristic point is that the result obtained in (16) differs from the expression on the left-hand side of (7) only by a constant factor. In effect, because $l_{e}^{\text {coll }}$ is a constant and $\tau_{e}=\Delta t$, the relation (16) between $\Delta E$ and $\Delta t$-considered with the accuracy to a constant coefficient-becomes much similar to that obtained in (7). By applying (16) in (7) we obtain the formula

$$
\frac{1}{2^{1 / 2}} m c l_{e}^{\text {coll }}>\hbar \text {. }
$$

A numerical check of validity of this relation is done in Section 3.

\section{Discussion}

The experimental result obtained for $l_{e}^{\text {coll }}$ in (16) is as follows $[7,8]$ :

$$
l_{e}^{\text {coll }} \approx 2 \AA=2 \times 10^{-8} \mathrm{~cm}
$$

The value can be subsequently substituted into (17). In view of the fundamental constants of nature used in the calculations we obtain

$$
\begin{aligned}
& \frac{1}{2^{1 / 2}} \times 9.1 \times 10^{-28} \times 3 \times 10^{10} \times 2 \times 10^{-8} \mathrm{ergsec} \\
& \approx 3 \times 10^{-25} \mathrm{ergsec} \\
& >\frac{6.62}{2 \pi} \times 10^{-27} \mathrm{ergsec} \approx 10^{-27} \mathrm{ergsec}
\end{aligned}
$$

which implies that relation (17) is satisfied, at least in the examined case.

Considering the relations between energy and time, there is, however, nothing special in the photoexcitation of a metal electron. Another well-known excitation can be, for instance, due to external electric field effect on the electrons. For very pure metals, the effect offers another mean free path of the electron than that considered in (17). This path, which is characteristic for the conduction process in a given metal, is due to electron interaction with the phonon medium combined with the medium of other electrons. The path length labelled by $l_{e}^{\text {cond }}$, is an equilibrium parameter for electron transport in the electric field, virtually independent of the field strength. As a result, instead of (17), we arrive at the formula:

$$
\frac{1}{2^{1 / 2}} m_{\mathrm{eff}} c l_{e}^{\mathrm{cond}}>\hbar
$$


Here $m_{\text {eff }}$ is the effective electron mass in the conductivity process.

As the tansport involves mainly the electrons close to the Fermi level, we have

$$
l_{e}^{\text {cond }}=v_{F} \tau_{e}^{\text {cond }}=\frac{\hbar k_{F}}{m_{\text {eff }}} \tau_{e}^{\text {cond }}
$$

where $\tau_{e}^{\text {cond }}$ is the relaxation time characteristic for conduction. The formula (21) transforms (20) into

$$
\frac{1}{2^{1 / 2}} m_{\mathrm{eff}} c \frac{\hbar k_{F}}{m_{\mathrm{eff}}} \tau_{e}^{\text {cond }}>\hbar
$$

equivalent to a simple relation

$$
\frac{1}{2^{1 / 2}} c k_{F} \tau_{e}^{\text {cond }}>1
$$

For $c=3 \times 10^{10} \mathrm{~cm} / \mathrm{s}, k_{F} \cong 10^{8} \mathrm{~cm}^{-1}$ and $\tau_{e}^{\text {cond }}=10^{-14} \div 10^{-15} \mathrm{~s}$ at room temperature, as valid for most metals [9], the relation (23) is obviously satisfied by the experimental data.

A separate estimate of $\tau_{e}^{\text {cond }}$ alone can be done on the basis of (3). This parameter is also assumed to approximate the decay time of the excited electron:

$$
\tau_{e}^{\text {cond }}=\Delta t
$$

For $k_{z}$ close to $k_{F}$ we can put

$$
k_{z}^{2}=\left(k_{F}-\Delta k_{z}\right)^{2} \cong k_{F}^{2}-2 k_{F} \Delta k_{z}
$$

where $\Delta k_{z}$ is considered to be a small fraction of the Bloch vector component $k_{z}$ :

$$
\Delta k_{\mathrm{z}}=\frac{2 \pi \Delta n_{\mathrm{z}}}{L}=\frac{2 \pi}{L} \ll k_{F}
$$

we have put $\Delta n_{z}=1$ and $L$ is the edge length of a cubic metal volume. As a result, the relation (3) becomes

$$
2 \frac{2 \pi}{L} k_{F} c^{2}(\Delta t)^{2}=\frac{4 \pi}{L} k_{F} c^{2}\left(\tau_{e}^{\text {cond }}\right)^{2}>1
$$

Therefore, we should have:

$$
\tau_{e}^{\text {cond }}>\frac{L^{1 / 2}}{(4 \pi)^{1 / 2} k_{F}^{1 / 2} C}
$$

For $L=1 \mathrm{~cm}$ and $k_{F} \approx 10^{8} \mathrm{~cm}^{-1}$ we obtain from (28) the condition

$$
\tau_{e}^{\mathrm{cond}}>10^{-15} \mathrm{~s}
$$

which is a number approaching rather perfectly the experimental value $\tau_{e}^{\text {cond }}$ for metals [9].

Let us note that the formula (1) which is in competition with (7) gives

$$
\tau_{e}^{\text {cond }}=\Delta t>\frac{\hbar}{\Delta E}
$$

Here

$$
\frac{\Delta E}{\hbar}=\frac{\hbar^{2}}{2 m} k_{F} \Delta k_{F} \frac{1}{\hbar}=\frac{\hbar}{2 m} k_{F} \Delta k_{z}=\frac{\hbar}{2 m} k_{F} \frac{2 \pi}{L}
$$

on condition the equality $\Delta k_{F}=\Delta k_{z}$ is applied for $\Delta k_{z}$ calculated in (26). A substitution of (28a) into (1a) gives

$$
\tau_{e}^{\text {cond }}>\frac{m L}{\pi \hbar k_{F}}=\frac{10^{-27} \times 1}{\pi \times 10^{-27} \times 10^{8}} \frac{\mathrm{g} \cdot \mathrm{cm}^{2}}{\mathrm{rg} \cdot \mathrm{sec}}=\frac{10^{-8}}{\pi} \mathrm{s}
$$

This result exceeds by many orders a typical experimental $\tau_{e}^{\text {cond }}$ in metals measured at normal conditions characterized by the room temperature; see [9] and (29).

A comparison of the Heisenberg relation (1) with the present one in (7) can be done also by considering an individual electron excitation due to the electric field effect. In this case from (1) and the experimental $\tau_{e}^{\text {cond }}$ put for $\Delta t$ we have

$$
\Delta E>\frac{\hbar}{\Delta t} \approx \frac{\hbar}{\tau_{e}^{\text {cond }}} \sim \frac{10^{-27}}{10^{-15}} \mathrm{erg} \cong 0.6 \mathrm{eV}
$$

which is an unrealistically high energy. On the other hand, formula (7) gives:

$$
\begin{aligned}
& \Delta E>\frac{\hbar^{2}}{2 m c^{2}(\Delta t)^{2}} \\
& =\frac{\hbar^{2}}{2 m c^{2}\left(\tau_{e}^{\text {cond }}\right)^{2}} \sim \frac{10^{-54}}{2 \times 10^{-27} \times 10^{21} \times 10^{-30}} \mathrm{erg} \\
& =\frac{1}{2} 10^{-18} \mathrm{erg} \cong \frac{1}{3} 10^{-6} \mathrm{eV}
\end{aligned}
$$

which is a much more reasonable value for an elementary excitation energy of an electron in the conduction process. In fact, a low excitation energy at the Fermi level is that entering (28a):

$$
\begin{aligned}
& \Delta E=\frac{\hbar^{2}}{2 m} k_{F} \Delta k_{F}=\frac{\hbar^{2}}{2 m} k_{F} \frac{2 \pi}{L} \\
& \cong 10^{-19} \pi \mathrm{erg} \cong 2 \times 10^{-7} \mathrm{eV}
\end{aligned}
$$

This is close to the result in (31).

\section{Conclusions}

The experimental results obtained for parameters related to the de-excitation of electrons in metals seem to favourite much more the relation (7) between $\Delta E$ and $\Delta t$ than the relation given in (1). A problem may arise, however, as to what extent the formula (7) can be useful in the case of non-free-electron transitions.

Another point concerns an agreement between (31) and (32). A so good agreement is probably an accidental since $\tau_{e}^{\text {cond }}$ is a parameter strongly dependent on the temperature $T$, especially at low $T$. For $T$ nearly 273 $\mathrm{K}$, as usually used in the presentation of the experimental data, the dependence of $\tau_{e}^{\text {cond }}$ on $T$ is rather weak. Consequently, the same dependence should apply to the 
mean free-electron path which tends to become an approximately constant parameter, in agreement with the behaviour of $l_{e}^{\text {coll }}$ discussed in Section 2 .

\section{REFERENCES}

[1] W. Heisenberg, "Ueber den Anschaulichen Inhalt der Quantentheoretischen Kinematik und Mechanik," Zeitschrift fuer Physik, Vol. 43, No. 3-4, 1927, pp. 172-198. doi:10.1007/BF01397280

[2] L. I. Schiff, "Quantum Mechanics," 3rd Edition, McGrawHill, New York, 1968.

[3] L. D. Landau and E. M. Lifshitz, "Quantum Mechanics," Pergamon, Oxford, 1965.

[4] M. Jammer, "The Philosophy of Quantum Mechanics," Wiley, New York, 1974.
[5] W. Schommers, "Space-Time and Quantum Phenomena," In: W. Schommers, Ed., Quantum Theory and Pictures of Reality, Springer, Berlin, 1989, pp. 217-277.

[6] S. Olszewski, "Magnetic Field Induction and Time Intervals of the Electron Transitions Approached on a Classical and Quantum-Mechanical Way," Journal of Modern Physics, Vol. 2, No. 11, 2011, pp. 1305-1309. doi:10.4236/jmp.2011.211161

[7] P. J. Vernier, "Photoemission," In: E. Wolf, Ed., Progress in Optics, Vol. 14, North-Holland, Amsterdam, 1976, pp. 245-325.

[8] N. V. Smith and G. B. Fischer, "Photoemission Studies of the Alkali Metals. II. Rubidium and Caesium," Physical Review B, Vol. 3, No. 11, 1971, pp. 3662-3670. doi:10.1103/PhysRevB.3.3662

[9] N. W. Ashcroft and N. D. Mermin, "Solid State Physics," Holt, Rinehart and Winston, New York, 1976. 